PROCEEDINGS OF THE

AMERICAN MATHEMATICAL SOCIETY

Volume 137, Number 10, October 2009, Pages 3227-3233

S 0002-9939(09)09913-4

Article electronically published on May 27, 2009

\title{
AFFINE ALGEBRAIC MONOIDS AS ENDOMORPHISMS' MONOIDS OF FINITE-DIMENSIONAL ALGEBRAS
}

\author{
ALEXANDER PEREPECHKO
}

(Communicated by Birge Huisgen-Zimmermann)

\begin{abstract}
We prove that any affine algebraic monoid can be obtained as the endomorphisms' monoid of a finite-dimensional (nonassociative) algebra.
\end{abstract}

\section{INTRODUCTION}

Let $\mathrm{K}$ be an algebraically closed field of arbitrary characteristic. Recall that an affine algebraic semigroup is an affine variety $M$ over $\mathrm{K}$ with an associative product $\mu: M \times M \rightarrow M$, which is a morphism of algebraic varieties. Denote an element $\mu(a, b)$ by $a b$. A semigroup is called a monoid if it contains an identity element $e \in M$ such that $e m=m e=m$ for any $m \in M$. An element $0 \in M$ is called zero if $0 m=m 0=0$ for any $m \in M$. Obviously, a monoid cannot contain more than one zero. It is well known that every affine algebraic monoid is isomorphic to a Zariski closed submonoid of the monoid $\mathrm{L}(V)$ of all linear operators on some finite-dimensional vector space $V$; e.g. see [4, Theorem 3.8] or [1, Lemma 1.11]. A systematic account of the theory of affine algebraic monoids is given in [3] and [4]. The classification of irreducible affine monoids, whose unit group is reductive, is obtained in [5] and [6].

Let $A$ be a finite-dimensional algebra over the field $\mathrm{K}$, i.e. a finite-dimensional vector space $A$ with a bilinear map $\alpha: A \times A \rightarrow A$. Note that the associativity or commutativity of the map $\alpha$ is not assumed. It is convenient to denote by $\operatorname{vect}(A)$ the underlying vector space of an algebra $A$. By an ideal of an algebra $A$ we mean a two-sided ideal. An algebra $A$ is called simple if it does not contain proper ideals. The set of all endomorphisms of $A$,

$$
\operatorname{End}(A):=\{\phi \in \mathrm{L}(\operatorname{vect}(A)) \mid \alpha(\phi(a), \phi(b))=\phi(\alpha(a, b)) \text { for } a, b \in A\},
$$

is a monoid with respect to composition. It is easy to check that this monoid is Zariski closed in $\mathrm{L}(\operatorname{vect}(A))$; therefore it is an affine algebraic monoid.

It is shown in 22 that any affine algebraic group can be realized as the group of automorphisms of some finite-dimensional simple algebra. This paper aims to obtain a similar realization of an arbitrary affine algebraic monoid $M$ as the endomorphisms' monoid of a finite-dimensional algebra $A$. In this case two differences occur. First, we cannot assume that $A$ is simple, since the kernel of any endomorphism is an ideal of $A$. Second, the monoid $\operatorname{End}(A)$ contains the zero $\mathfrak{z} \in \operatorname{End}(A)$,

Received by the editors September 13, 2008.

2000 Mathematics Subject Classification. Primary 17A36, 20M20; Secondary 16W22, 20 G20.

(C)2009 American Mathematical Society 
$\mathfrak{z}(a)=0$ for any $a \in A$, while $M$ does not necessarily contain a zero. Under these circumstances we obtain the following result.

Theorem 1.1. For any affine algebraic monoid $M$ there exists a finite-dimensional algebra $A$ such that $\operatorname{End}(A) \cong M \sqcup\{\mathfrak{z}\}$, where $\{\mathfrak{z}\}$ is an (isolated) component of the monoid $\operatorname{End}(A)$.

Particularly, if $M$ is an affine algebraic group, then there exists an algebra $A$ such that $\operatorname{Aut}(A) \cong M($ see 2$)$.

Example 1.2. Let us consider the monoid $M=\mathrm{L}(V)$ for a finite-dimensional space $V$. Then we may take the algebra $A$ constructed in the following way. First, let $e$ be a left identity of $A$ and

$$
\operatorname{vect}(A):=\langle e\rangle \oplus V,
$$

where $\langle X\rangle$ stands for the linear span of a set $X$. Next, for any $v, w \in V$ put $\alpha(v, w)=0, \alpha(v, e)=\lambda v$, where $\lambda \in \mathrm{K} \backslash\{0,1\}$. Taking into account equations $\alpha(e, v)=v$ and $\alpha(e, e)=e$, we obtain the multiplication table for $A$.

Note that any endomorphism sends $e$ to $e$ or 0 , since these two are the only idempotents of $A$. This way, the reader will easily prove that $\operatorname{End}(A) \cong \mathrm{L}(V) \sqcup\{\mathfrak{z}\}$.

Example 1.3. Assume char $\mathrm{K} \neq 2$. Consider a two-dimensional space $V$ over $\mathrm{K}$ with a basis $\left\{v_{1}, v_{2}\right\}$ and the exterior algebra $\Lambda(V)$ with a basis $\left\{1, v_{1}, v_{2}, v_{1} \wedge v_{2}\right\}$. Let us take a monoid $M \subset \mathrm{L}(\operatorname{vect}(\Lambda(V)))$,

$$
M:=\left\{\left(\begin{array}{cccc}
1 & 0 & 0 & 0 \\
0 & b_{11} & b_{12} & 0 \\
0 & b_{21} & b_{22} & 0 \\
0 & c_{1} & c_{2} & d
\end{array}\right) \mid d=\operatorname{det}\left(\begin{array}{cc}
b_{11} & b_{12} \\
b_{21} & b_{22}
\end{array}\right), b_{i j}, c_{i} \in \mathrm{K}\right\} .
$$

One may prove that $M$ acts on $\Lambda(V)$ by endomorphisms. Moreover, $\operatorname{End}(\Lambda(V))=$ $M \sqcup\{\mathfrak{z}\}$. Generally, a similar equation holds for the exterior algebra of an arbitrary space.

The proof of Theorem 1.1 consists of two steps. First, for every finite-dimensional space $U$ and its subspace $S$ we construct a finite-dimensional algebra $A$ such that $\operatorname{End}(A)$ is isomorphic to $\mathrm{L}(U)_{S} \sqcup\{\mathfrak{z}\}$, where $\mathrm{L}(U)_{S}$ is the normalizer of some vector subspace $S$ of a special L $(U)$-module. Second, an arbitrary affine algebraic monoid $M$ is represented as $\mathrm{L}(U)_{S}$ for appropriate $U$ and $S$. Overall, we follow the scheme of the proof in 2, but the ideas of the first step are significantly changed.

\section{Some SPECIAL ALGEBRAS}

In this section we define and study some finite-dimensional algebras to be used hereafter.

2.1. Algebra $A(V, S)$. Let $V$ be a nonzero finite-dimensional vector space. Denote by $\mathrm{T}(V)$ the tensor algebra of $V$ and by $\mathrm{T}(V)_{+}$its maximal homogeneous ideal,

$$
\mathrm{T}(V)_{+}:=\bigoplus_{i \geqslant 1} V^{\otimes i}
$$

endowed with the natural $\mathrm{L}(V)$-structure

$$
g \cdot t_{i}:=g^{\otimes i}\left(t_{i}\right), \quad g \in \mathrm{L}(V), t_{i} \in V^{\otimes i} .
$$

Thus, $\mathrm{L}(V)$ acts on $\mathrm{T}(V)_{+}$faithfully by endomorphisms. Therefore we may identify $\mathrm{L}(V)$ with the corresponding submonoid of $\operatorname{End}\left(\mathrm{T}(V)_{+}\right)$. 
Fix an integer $r>1$. For an arbitrary subspace $S \subseteq V^{\otimes r}$ we define

$$
I(S):=S \oplus\left(\bigoplus_{i>r} V^{\otimes i}\right) .
$$

It is an ideal of $\mathrm{T}(V)_{+}$. Define $A(V, S)$ as the factor algebra modulo this ideal,

$$
A(V, S):=\mathrm{T}(V)_{+} / I(S) .
$$

Then

$$
\operatorname{vect}(A(V, S))=\left(\bigoplus_{i=1}^{r-1} V^{\otimes i}\right) \oplus\left(V^{\otimes r} / S\right) .
$$

We may consider $\mathrm{L}(V)_{S}:=\{\phi \in \mathrm{L}(V) \mid \phi(S) \subseteq S\} \subset \mathrm{L}(V)$.

Proposition 2.1. $\{\sigma \in \operatorname{End}(A(V, S)) \mid \sigma(V) \subseteq V\}=\mathrm{L}(V)_{S}$.

Proof. By definition, elements of $A(V, S)$ are equivalence classes $x+I(S), x \in$ $\mathrm{T}(V)_{+}$. Let us prove the inclusion $\subseteq$. Consider $\sigma \in \operatorname{End}(A(V, S))$ such that $\sigma(V) \subseteq V$. Then the $\sigma$-action coincides with the action of $\widetilde{\sigma}:=\left.\sigma\right|_{V} \in \mathrm{L}(V)$ on $A(V, S)$ in accordance with (2.2), since the algebra $A(V, S)$ is generated by $V$. The $\sigma$-action preserves the zero of $A(V, S)$; hence $\widetilde{\sigma}(I(S)) \subseteq I(S)$ and $\sigma \in \mathrm{L}(V)_{S}$.

Now we prove the inverse inclusion. For arbitrary subsets $X, Y \subset \mathrm{T}(V)$ define $X \otimes Y:=\{x \otimes y \mid x \in X, y \in Y\} \subset \mathrm{T}(V)$. Let $\sigma \in \mathrm{L}(V)_{S}$. Then $\sigma((x+I(S)) \otimes$ $(y+I(S))) \subseteq \sigma(x \otimes y)+I(S)=\sigma(x) \otimes \sigma(y)+I(S)$ by definition of the $\mathrm{L}(V)$-action on $\mathrm{T}(V)_{+}$. Hence $\sigma \in \operatorname{End}(A(V, S))$.

\subsection{Algebra $D(P, U, S, \gamma)$.}

Lemma 2.2. Let $A$ be an algebra with a left identity $e \in A$ such that $\operatorname{vect}(A)=$ $\langle e\rangle \oplus A_{1} \oplus \cdots \oplus A_{r}$, where $A_{i}$ is the eigenspace with an eigenvalue $\alpha_{i} \neq 0,1$ for the operator of right multiplication of $A$ by $e$. Assume that 0 and $e$ are the only idempotents in A. Then

(i) $e$ is the unique left identity in $A$;

(ii) if $\sigma \in \operatorname{End}(A)$, then either $\sigma(e)=e$ and $\sigma\left(A_{i}\right) \subseteq A_{i}$ for any $i$, or $\sigma=\mathfrak{z}$.

Proof. (i) The left identity is a nonzero idempotent. Hence it is unique.

(ii) Since the image of an idempotent is an idempotent, $\sigma(e)=0$ or $\sigma(e)=e$. If $\sigma(e)=0$, then $\sigma(a)=\sigma(e a)=\sigma(e) \sigma(a)=0$, i.e. $\sigma=\mathfrak{z}$. Now assume that $\sigma(e)=e$. Then $\sigma\left(A_{i}\right)$ is the eigenspace with an eigenvalue $\alpha_{i} \neq 0,1$ for the operator of right multiplication by $e$. Hence $\sigma\left(A_{i}\right) \subseteq A_{i}$.

Let $P$ be a two-dimensional vector space with a basis $\left\{p_{1}, p_{2}\right\}, U$ be a nonzero finite-dimensional space, and

$$
V:=P \oplus U
$$

Fix an integer $r>1$ as well as

(i) a subspace $S \subset V^{\otimes r}$;

(ii) a sequence $\gamma=\left(\gamma_{1}, \ldots, \gamma_{6}\right) \in(\mathrm{K} \backslash\{0,1\})^{6}, \gamma_{i} \neq \gamma_{j}$ for $i \neq j$.

Define an algebra $D(P, U, S, \gamma)$ in the following way. First, $A(V, S)$ is the subalgebra of $D(P, U, S, \gamma)$ and elements $b, c, d, e \in D(P, U, S, \gamma)$ are such that

$$
\operatorname{vect}(D(P, U, S, \gamma))=\langle e\rangle \oplus\langle b\rangle \oplus\langle c\rangle \oplus\langle d\rangle \oplus \operatorname{vect}(A(V, S)) .
$$

Second, the following conditions hold:

(D1) $e$ is the left identity of $D(P, U, S, \gamma)$. 
(D2) $\langle b\rangle,\langle c\rangle,\langle d\rangle$ as well as $P, U \subset V=V^{\otimes 1} \subset A(V, S)$ and $\left(\bigoplus_{i=2}^{r-1} V^{\otimes i}\right) \oplus$ $\left(V^{\otimes r} / S\right) \subset A(V, S)$ are the eigenspaces with the eigenvalues $\gamma_{1}, \ldots, \gamma_{6}$, respectively, of the operator of right multiplication by $e$.

(D3) The multiplication table for $b, c, d$ is

$$
\begin{array}{lll}
b \cdot b:=0, & b \cdot c:=c+\gamma_{b c} b, & b \cdot d:=0, \\
c \cdot b:=-c, & c \cdot c:=b, & c \cdot d:=e, \\
d \cdot b:=p_{1}, & d \cdot c:=d, & d \cdot d:=p_{2},
\end{array}
$$

where $\gamma_{b c}=\frac{\gamma_{2}-\gamma_{1}}{\gamma_{2}-\gamma_{3}}$.

(D4) $\langle b, c, d\rangle \cdot A(V, S)=A(V, S) \cdot\langle b, c, d\rangle=0$.

Define the action of $g \in \mathrm{L}(V)_{S}$ on $\operatorname{vect}(D(P, U, S, \gamma))$ as follows: $\left.g\right|_{\langle b\rangle}=\left.g\right|_{\langle c\rangle}=$ $\left.g\right|_{\langle d\rangle}=\left.g\right|_{\langle e\rangle}=$ id, $\left.g\right|_{V}$ is the natural $\mathrm{L}(V)$-action on $V$, and on other summands of $A(V, S)$ it is defined by (2.2). By Proposition 2.1 we may identify $\mathrm{L}(V)_{S}$ with the corresponding submonoid of $\mathrm{L}(\operatorname{vect}(D(P, U, S, \gamma)))$. Further, we may consider an embedding $\mathrm{L}(U) \hookrightarrow \mathrm{L}(V),\left.h \mapsto \mathrm{id}\right|_{P} \oplus h$. Thus, $\mathrm{L}(U)_{S} \subseteq \mathrm{L}(V)_{S}$, and we obtain the $\mathrm{L}(U)_{S}$-action on $\operatorname{vect}(D(P, U, S, \gamma))$.

Proposition 2.3. We have

$$
\operatorname{End}(D(P, U, S, \gamma))=\mathrm{L}(U)_{S} \sqcup\{\mathfrak{z}\},
$$

where $\{\mathfrak{z}\}$ is an (isolated) component of the monoid $\operatorname{End}(D(P, U, S, \gamma))$.

Proof. First of all, we show that 0 and $e$ are the only idempotents of $D(P, U, S, \gamma)$. Indeed, let $\varepsilon=\lambda_{e} e+\lambda_{b} b+\lambda_{c} c+\lambda_{d} d+a$, where $a \in A(V, S)$. Then

$$
\begin{aligned}
& \varepsilon^{2}=\left(\lambda_{e}^{2}+\right.\left.\lambda_{c} \lambda_{d}\right) e+\left(\lambda_{b} \lambda_{e}\left(1+\gamma_{1}\right)+\lambda_{c}^{2}+\lambda_{b} \lambda_{c} \gamma_{b c}\right) b \\
&+\lambda_{c} \lambda_{e}\left(1+\gamma_{2}\right) c+\left(\left(1+\gamma_{3}\right) \lambda_{d} \lambda_{e}+\lambda_{d} \lambda_{c}\right) d+a^{\prime} \\
&=\lambda_{1} e+\lambda_{2} d+\lambda_{3} c+\lambda_{4} b+a, \text { where } a, a^{\prime} \in A(V, S) .
\end{aligned}
$$

Hence

$$
\begin{gathered}
\lambda_{e}=\lambda_{e}^{2}+\lambda_{c} \lambda_{d}, \\
\lambda_{b}=\lambda_{b} \lambda_{e}\left(1+\gamma_{1}\right)+\lambda_{c}^{2}+\lambda_{b} \lambda_{c} \gamma_{b c}, \\
\lambda_{c}=\lambda_{c} \lambda_{e}\left(1+\gamma_{2}\right), \\
\lambda_{d}=\lambda_{d} \lambda_{e}\left(1+\gamma_{3}\right)+\lambda_{c} \lambda_{d} .
\end{gathered}
$$

Assume $\lambda_{c} \neq 0$. By (2.12) $, 1+\gamma_{2} \neq 0, \lambda_{e}=\frac{1}{1+\gamma_{2}}$ and $\lambda_{c} \lambda_{d}=\lambda_{e}-\lambda_{e}^{2} \neq 0$, so $\lambda_{d} \neq 0$. Hence equation (2.13) implies $\lambda_{c}=1-\lambda_{e}\left(1+\gamma_{3}\right)=\frac{\gamma_{2}-\gamma_{3}}{1+\gamma_{2}}$. Finally, by (2.11) we have $\lambda_{c}^{2}=\lambda_{b}\left(1-\lambda_{e}\left(1+\gamma_{1}\right)-\lambda_{c} \gamma_{b c}\right)=\lambda_{b}\left(\frac{\gamma_{2}-\gamma_{1}}{1+\gamma_{2}}-\frac{\gamma_{2}-\gamma_{3}}{1+\gamma_{2}} \cdot \frac{\gamma_{2}-\gamma_{1}}{\gamma_{2}-\gamma_{3}}\right)=0$. From this contradiction we deduce $\lambda_{c}=0$.

Moreover, $\lambda_{e}=0$ or $\lambda_{e}=1$ by (2.10). If $\lambda_{e}=0$, then $\lambda_{b}=\lambda_{d}=0, \varepsilon=$ $a \in A(V, S)$ and $\varepsilon=0$, since zero is the only idempotent of $A(V, S)$. Now assume $\lambda_{e}=1$. From equations (2.11) and (2.13) accordingly follow $\lambda_{b}=0$ and $\lambda_{d}=0$. Thus, $\varepsilon=e+a, a \in A(V, S)$.

Let $a=a_{P}+a_{U}+a_{\Sigma}$, where $a_{P} \in P, a_{U} \in U, a_{\Sigma} \in\left(\bigoplus_{i=2}^{r-1} V^{\otimes i}\right) \oplus\left(V^{\otimes r} / S\right)$. Then

$$
\varepsilon^{2}=e+\left(1+\gamma_{4}\right) a_{P}+\left(1+\gamma_{5}\right) a_{U}+a_{\Sigma}^{\prime}=e+a_{P}+a_{U}+a_{\Sigma},
$$


where $a_{\Sigma}^{\prime} \in\left(\bigoplus_{i=2}^{r-1} V^{\otimes i}\right) \oplus\left(V^{\otimes r} / S\right)$. Hence $a_{U}=a_{P}=0$. Assume $a_{\Sigma} \neq 0$. Then we may write $a_{\Sigma}=a_{k}+\ldots+a_{r}, a_{k} \neq 0$, where $a_{i} \in V^{\otimes i}$ for $i<r$ and $a_{r} \in V^{\otimes r} / S$. This way,

$$
\left(e+a_{k}+\ldots+a_{r}\right)^{2}=e+\left(1+\gamma_{6}\right) a_{k}+a^{\prime \prime}=e+a_{k}+\ldots+a_{r},
$$

where $a^{\prime \prime} \in\left(\bigoplus_{i=k+1}^{r-1} V^{\otimes i}\right) \oplus\left(V^{\otimes r} / S\right)$ for $k<r$ and $a^{\prime \prime}=0$ for $k=r$. This implies $a_{k}=0$, a contradiction. Hence $a_{\Sigma}=0$ and $\varepsilon=e$.

Thus, $D(P, U, S, \gamma)$ contains no idempotents different from 0 and $e$. Let $\sigma \in$ $\operatorname{End}(D(P, U, S, \gamma)) \backslash\{\mathfrak{z}\}$. By Lemma 2.2, $\sigma(e)=e$ and $\langle b\rangle,\langle c\rangle,\langle d\rangle, P, U, A(V, S)$ are $\sigma$-invariant. Let $\sigma(b)=\delta_{b} b, \sigma(c)=\delta_{c} c, \sigma(d)=\delta_{d} d$. The equations $c d=$ $e, d c=d, c b=-c$ imply $\delta_{c} \delta_{d}=1, \delta_{c} \delta_{d}=\delta_{d}, \delta_{b} \delta_{c}=\delta_{c}$. One may check that $\delta_{b}=\delta_{c}=\delta_{d}=1$. Finally, the equations $d b=p_{1}$, $d d=p_{2}$ imply $\left.\sigma\right|_{P}=\operatorname{id}_{P}$.

Since $V$ and $A(V, S)$ are $\sigma$-invariant, $\left.\sigma\right|_{A(V, S)} \in \mathrm{L}(V)_{S}$ by Proposition 2.1. Taking into account $\left.\sigma\right|_{P}=\operatorname{id}_{P}$ and $\sigma(U) \subseteq U$, we obtain $\sigma \in \mathrm{L}(U)_{S}$.

\section{AfFine MONOIDS AS THE NORMALIZERS OF LINEAR SUBSPACES}

Proposition 3.1. Let $M$ be an affine algebraic monoid. There is a finite-dimensional vector space $U$ and an integer $r>1$ such that the following holds. Let $P$ be a two-dimensional vector space with a trivial $\mathrm{L}(U)$-action. Then the $\mathrm{L}(U)$-module $(P \oplus U)^{\otimes r}$ contains a linear subspace $S$ such that $\mathrm{L}(U)_{S} \cong M$.

Proof. Since there exists a closed embedding $M \hookrightarrow \mathrm{L}(U)$ for some finite-dimensional space $U$, we may suppose $M \subseteq \mathrm{L}(U)$. Consider the action of $\mathrm{L}(U)$ on itself by left multiplication. Additionally, consider the $\mathrm{L}(U)$-action on the algebra $\mathrm{K}[\mathrm{L}(U)]$ of regular functions on $\mathrm{L}(U)$,

$$
(g \cdot f)(u):=f(u g), \quad g, u \in \mathrm{L}(U), f \in \mathrm{K}[\mathrm{L}(U)] .
$$

Denote $d:=\operatorname{dim} U$. Note that the $\mathrm{L}(U)$-modules $\mathrm{K}[\mathrm{L}(U)]$ and $\operatorname{Sym}\left(U^{\oplus d}\right)$ are isomorphic. To prove this, it suffices to associate a linear function on $\mathrm{L}(U)$ to every vector $\left(u_{1}, \ldots, u_{d}\right) \in U^{\oplus d}$, since $\mathrm{K}[\mathrm{L}(U)]=\operatorname{Sym}\left(\mathrm{L}(U)^{*}\right)$. Identify $U$ with $\mathrm{K}^{d}, \mathrm{~L}(U)$ with $\operatorname{Mat}_{d \times d}(\mathrm{~K})$; let $A$ be in $\mathrm{L}(U), B$ be a matrix with columns $u_{1}, \ldots, u_{d}$. Set $l_{u_{1}, \ldots, u_{d}}(A):=\operatorname{tr} A B$. Then $\left(g \cdot l_{u_{1}, \ldots, u_{d}}\right)(A)=\operatorname{tr} A g B=l_{g u_{1}, \ldots, g u_{d}}(A)$; i.e. we have an $\mathrm{L}(U)$-equivariant isomorphism.

By the definition of a symmetric algebra fix a natural epimorphism

$$
\xi: \mathrm{T}\left(U^{\oplus d}\right) \rightarrow \operatorname{Sym}\left(U^{\oplus d}\right) \cong \mathrm{K}[\mathrm{L}(U)] .
$$

There is a finite-dimensional subspace $W \subset \mathrm{K}[\mathrm{L}(U)]$ such that

$$
\mathrm{L}(U)_{W}=M \text {. }
$$

In order to prove this, one may show that a linear span of an $\mathrm{L}(U)$-'orbit' of an arbitrary function $f \in \mathrm{K}[\mathrm{L}(U)]$ is finite-dimensional. Indeed, since the $\mathrm{L}(U)$-action is a morphism, $(g \cdot f)(u)=f(u g) \in \mathrm{K}[\mathrm{L}(U) \times \mathrm{L}(U)]=\mathrm{K}[\mathrm{L}(U)] \otimes \mathrm{K}[\mathrm{L}(U)]$, where $u, g \in \mathrm{L}(U)$, there are functions $F_{j}, H_{j} \in \mathrm{K}[\mathrm{L}(U)]$ such that

$$
(g \cdot f)(u)=\sum_{j=1}^{n} F_{j}(u) H_{j}(g) .
$$

Therefore, the $\mathrm{L}(U)$-'orbit' of the function $f$ is contained in the finite-dimensional subspace $\left\langle F_{1}, \ldots, F_{n}\right\rangle$. 
Let $I(M)=\left(f_{1}, \ldots, f_{t}\right) \triangleleft \mathrm{K}[\mathrm{L}(U)]$ be the ideal of functions vanishing on $M$. Summing the linear spans of $\mathrm{L}(U)$-'orbits' of the functions $f_{i}$ we obtain a finitedimensional $\mathrm{L}(U)$-invariant subspace $V \subset \mathrm{K}[\mathrm{L}(U)]$. Define $W=I(M) \cap V$. First, it contains $f_{1}, \ldots, f_{t}$. Second, it is $M$-invariant, since the ideal $I(M)$ is $M$-invariant. Obviously, $g \in M$ implies $g \cdot W \subseteq W$. On the other hand, suppose that $g \cdot W \subseteq W$, where $g \in \mathrm{L}(U)$. Then $f_{i}(g)=\left(g \cdot f_{i}\right)(E)=0$ for $i=1, \ldots, t$, where $E$ is the identity of $\mathrm{L}(U)$ and is automatically contained in $M$. Therefore, $g \in M$. This proves (3.3).

Further, since the space $W$ is finite-dimensional, there is an integer $h \in \mathbb{Z}_{+}$such that

$$
W \subseteq \xi\left(\bigoplus_{i \leqslant h}\left(U^{\oplus d}\right)^{\otimes i}\right) .
$$

Define $W^{\prime}:=\xi^{-1}(W) \cap\left(\bigoplus_{i \leqslant h}\left(U^{\oplus d}\right)^{\otimes i}\right)$. The $\mathrm{L}(U)$-equivariance of $\xi$ implies

$$
\mathrm{L}(U)_{W^{\prime}}=\mathrm{L}(U)_{W} .
$$

Fix a basis $\left\{p_{1}, p_{2}\right\}$ of the space $P$. There exists an embedding of $\mathrm{L}(U)$-modules

$$
\iota: \mathrm{T}\left(U^{\oplus d}\right) \hookrightarrow \mathrm{T}\left(\left\langle p_{1}\right\rangle \oplus U\right) .
$$

Indeed, let $U_{i}$ be the $i$ th summand of $U^{\oplus d}$. Consider an arbitrary basis $\left\{f_{i j} \mid j=\right.$ $1, \ldots, d\}$ of $U_{i}$ and define an embedding as follows:

$$
\iota\left(f_{i_{1} j_{1}} \otimes \ldots \otimes f_{i_{t} j_{t}}\right):=p_{1}^{\otimes i_{1}} \otimes f_{i_{1} j_{1}}^{\prime} \otimes \ldots \otimes p_{1}^{\otimes i_{t}} \otimes f_{i_{t} j_{t}}^{\prime},
$$

where $f_{i j}^{\prime}$ is the image of $f_{i j}$ under the identity isomorphism $U_{i} \rightarrow U$. It is easy to check that the embedding $\iota: \mathrm{T}\left(U^{\oplus d}\right) \rightarrow \mathrm{T}\left(\left\langle p_{1}\right\rangle \oplus U\right)$ defined on the basis of $\mathrm{T}\left(U^{\oplus d}\right)_{+}$by formula (3.8) and sending 1 to 1 is the one required.

Now we may consider a space $W^{\prime \prime}:=\iota\left(W^{\prime}\right)$,

$$
\mathrm{L}(U)_{W^{\prime \prime}}=\mathrm{L}(U)_{W^{\prime}} .
$$

Since $W^{\prime \prime}$ is finite-dimensional, there exists an integer $b \in \mathbb{N}$ such that

$$
W^{\prime \prime} \subseteq \bigoplus_{i \leqslant b}\left(\left\langle p_{1}\right\rangle \oplus U\right)^{\otimes i} .
$$

Take $r \geqslant b$ such that $r>1$ and consider a linear mapping

$$
\iota_{r}: \bigoplus_{i \leqslant b}\left(\left\langle p_{1}\right\rangle \oplus U\right)^{\otimes i} \rightarrow(P \oplus U)^{\otimes r}, \quad f_{i} \mapsto p_{2}^{\otimes(r-i)} \otimes f_{i}, f_{i} \in\left(\left\langle p_{1}\right\rangle \oplus U\right)^{\otimes i} .
$$

Obviously, $\iota_{r}$ is an embedding of $\mathrm{L}(U)$-modules. Define $S=\iota_{r}\left(W^{\prime \prime}\right)$. Then

$$
\mathrm{L}(U)_{S}=\mathrm{L}(U)_{W^{\prime \prime}} .
$$

Now the claim follows from equations (3.3), (3.6), (3.9) and (3.12).

Proof of Theorem 1.1, Let $M$ be an arbitrary affine algebraic monoid, $U, b, r, P, S$ be as in Proposition 3.1. Fix some set $\gamma \in(\mathrm{K} \backslash\{0,1\})^{6}$ such that $\gamma_{i} \neq \gamma_{j}$ for $i \neq j$, and consider the algebra $D(P, U, S, \gamma)$. It follows from Proposition 3.1 and Proposition 2.3 that $\operatorname{End}(D(P, U, S, \gamma)) \cong M \sqcup\{\mathfrak{z}\}$.

\section{ACKNOWLEDGEMENT}

The author expresses his sincere thanks to I.V. Arzhantsev for posing the problem and for useful discussions. 


\section{REFERENCES}

[1] I.V. Arzhantsev, Affine embeddings of homogeneous spaces, in "Surveys in Geometry and Number Theory", N. Young (Editor), London Math. Soc. Lecture Notes Series 338, Cambridge Univ. Press, Cambridge, 2007, 1-51. MR2306139(2008d:14074)

[2] N.L. Gordeev and V.L. Popov, Automorphism groups of finite dimensional simple algebras, Annals of Mathematics 158 (2003), 1041-1065. MR2031860 (2005b:20086)

[3] M.S. Putcha, Linear algebraic monoids, London Math. Soc. Lecture Notes Series 133, Cambridge Univ. Press, Cambridge, 1988. MR.964690 (90a:20003)

[4] L. Renner, Linear algebraic monoids, Encyclopaedia of Mathematical Sciences 134, SpringerVerlag, Berlin-Heidelberg, 2005. MR 2134980 (2006a:20002)

[5] A. Rittatore, Algebraic monoids and affine embeddings, Transform. Groups 3 (1998), no. 4, 375-396. MR1657536 (2000a:14056)

[6] E.B. Vinberg, On reductive algebraic semigroups, Amer. Math. Soc. Transl. (2) 169 (1995), 145-182. MR.1364458 (97d:20057)

Department of Higher Algebra, Faculty of Mechanics and Mathematics, Moscow State University, Leninskie Gory, Moscow, 119991, Russia

E-mail address: perepechko@mccme.ru 